\title{
THERMOMECHANICS OF PHASE TRANSITIONS IN CLASSICAL FIELD THEORY
}


This page is intentionally left blank 


\section{THERMOMECHANICS \\ OF PHASE TRANSITIONS \\ IN CLASSICAL \\ FIELD THEORY}

\section{Antonio Romano}

Dipartimento di Matematica e Applicazioni

Università degli Studi di Napoli "Federico II" 
Published by

World Scientific Publishing Co. Pte. Ltd.

P O Box 128, Farrer Road, Singapore 9128

USA office: Suite 1B, 1060 Main Street, River Edge, NJ 07661

UK office: 73 Lynton Mead, Totteridge, London N20 8DH

\section{Library of Congress Cataloging-in-Publication Data \\ Romano, Antonio.}

Thermomechanics of phase transitions in classical field theory/ Antonio Romano.

p. $\quad \mathrm{cm}$.-- (Series on advances in mathematics for applied sciences ; vol. 13.)

Includes bibliographical references.

ISBN 9810213980

1. Phase transformations (Statistical physics) 2. Surfaces

(Physics) 3. Field theory (Physics) 4. Continuum mechanics.

I. Title. II. Series.

QC175.16.P5R65 1993

530.4'74--dc20

93-30499

CIP

Copyright (C) 1993 by World Scientific Publishing Co. Pte. Ltd.

All rights reserved. This book, or parts thereof, may not be reproduced in any form or by any means, electronic or mechanical, including photocopying, recording or any information storage and retrieval system now known or to be invented, without written permission from the Publisher.

For photocopying of material in this volume, please pay a copying fee through the Copyright Clearance Center, Inc., 27 Congress Street, Salem, MA 01970, USA.

Printed in Singapore by JBW Printers \& Binders Pte. Ltd. 


\section{PREFACE}

The phase transitions in a substance $C$ are due to certain weak interactions among the molecules of $\mathcal{C}$ which might correctly be described by Quantum Mechanics. However, if we abstain from understanding every minute detail of behaviours in phase transitions, we can limit ourselves to describe these phenomena by resorting to much simpler and tractable schemes. In other words, we use a macroscopic description which is unable to explain either why a state change takes place or which modifications this produces at a microscopic level.

The first approach starts from the experimental observation that the different phases are separated by very narrow layers across which the fields associated to $C$ vary continuously but sharply. Owing to the high values assumed by the gradients of the fields in these layers, the ordinary constitutive equations are supposed to depend weakly on the higher order gradients of the aforesaid fields. This, in turn, implies that the highest order derivatives in the local equations of balance are multiplied by small coefficients. As is well-known, this property leads just to boundary layers whose localization and forms depend on the equations as well as on the region occupied by $\mathcal{C}$ (see for instance $[50,51]$ ). 
Another approach to attain a macroscopic description of phase transitions is obtained by resorting to the models of continua with an interface. The basic idea of this approach is that we can substitute the narrow layers between the phases with surfaces of discontinuity for the volume fields provided that we associate to these surfaces some physical attributes which evoke the complex structure of the fields in the layers they substitute. This essay is just dedicated to this approach without any attempt to compare it with the alternative approach of boundary layers (see for instance [52] for a comparison).

After some kinematical preliminaries (chapters 1 and 2), the detailed analysis of the balance equations for a system with an interface as well as of the average procedures which allow to define the physical attributes to associate to the interface are discussed in chapter 3 . The remaining chapters are dedicated to the applications of the general scheme to the equilibrium and thermodynamical evolution of phase transitions in liquids, solids, mixtures, crystals and ferroelectric materials. For the phase transitions in these last two materials a suitable use of the nonlocal theories of Continuum Mechanics is suggested. 


\section{CONTENTS}

PREFACE

\section{Chapter 1: GEOMETRY OF SURFACES}

1.1 Definitions of regular surfaces, holonomic bases and metrics........ 1

1.2 The second fundamental form and the Gauss-Weingarten equations.......................................................................... 5

1.3 Normal curvature ........................................................... 9

1.4 Elliptic, hyperbolic and parabolic points. Mean and Gaussian curvatures

1.5 Lines of curvature ........................................................... 14

1.6 Surface gradient operator and Gauss theorem....................... 16

\section{Chapter 2: KINEMATICS OF SURFACES}

2.1 Velocity of a moving surface

2.2 The Thomas derivative .................................................. 28

2.3 The velocity of a moving curve on a moving surface............... 30 
viii PHASE TRANSITIONS IN CLASSICAL FIELD THEORY

2.4 Time derivative of a volume integral................................. 31

2.5 Time derivative of a surface integral................................... 34

2.6 Two time derivation formulas derived from (2.39) and (2.44)... 38

\section{Chapter 3: BALANCE LAWS FOR A CONTINUOUS SYSTEM WITH AN INTERFACE}

3.1 General balance laws 42

3.2 Mechanical modelling of nonmaterial interfaces ..................... 48

3.3 Balance equations for a system with an interface ................... 58

3.4 The reduced dissipation inequalities .................................. 65

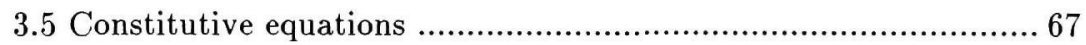

\section{Chapter 4: PHASE EQUILIBRIUM}

4.1 Phase equilibrium boundary value problems ......................... 73

4.2 Equilibrium of fluid phases separated by a planar interface in the absence of body force............................................................. 76

$4.3 \mathrm{~A}$ brief résumé of phenomenology of state changes.................... 78

4.4 Equilibrium of fluid phases separated by nonplanar interfaces.... 84 
4.5 Equilibrium of fluid phases separated by spherical interfaces 86

4.6 Equilibrium between isotropic solid and fluid phases 93

4.7 Variational formulation of phase equilibrium 97

\section{Chapter 5: STATIONARY AND TIME DEPENDENT PROBLEMS}

5.1 A stationary problem and its nondimensional analysis 105

5.2 On the approximate evolution of solid-liquid state changes 112

5.3 On the approximate evolution of liquid-vapour state changes.....119

5.4 The case of a perfect gas.

124

\section{Chapter 6: PHASE CHANGES IN MIXTURES}

6.1 Balance laws in classical mixtures

6.2 Constitutive equations and dissipation inequalities...................134

6.3 Phase equilibrium in a binary fluid mixture ............................140

6.4 The influence of mass adsorption on surface tension................ 143

6.5 The Gibbs principle for phase equilibrium in fluid mixtures.......147

6.6 The evaporation of a fluid into a gas..................................... 149 


\section{Chapter 7: CRYSTAL GROWTH}

7.1 Gibbs' and Wulff's equilibrium laws.................................... 159

7.2 About Wulff's construction........................................... 166

7.3 An introduction to nonlocal thermomechanical theory...............169

7.4 Nonlocal balance laws......................................................... 173

7.5 Nonlocal reduced dissipation inequalities............................... 181

7.6 Preliminary considerations on crystal growth....................... 183

7.7 A mathematical model of crystal growth in a binary nonreacting

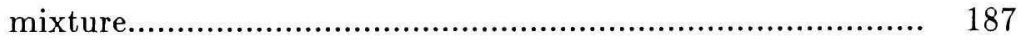

7.8 On the crystal equilibrium.............................................. 194

7.9 The energy equation and the reduced dissipation inequality........199

7.10 The free boundary value problem describing the crystal

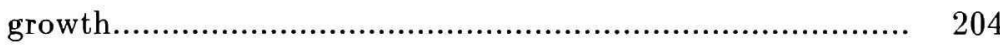

\section{Chapter 8: SYSTEMS WITH INTERFACES AND FERROELECTRICITY}

8.1 A brief survey of ferroelectricity................................... 208

8.2 Maxwell's equations for a moving continuous system...............211

8.3 A system of a rigid ferroelectric crystal in the presence of conductors and quasi-static approximation............................213

8.4 The balance of energy and the entropy inequality...................221

8.5 Constitutive equations and thermodynamical restrictions..........224 
8.6 Structure of the Weiss domains........................................... 228

8.7 The boundary value problem for the Weiss domains................234

8.8 Weiss' domains in the absence of electric field at equilibrium.............................................................. 236

\section{REFERENCES}

